\title{
Robotic Fish Technology and Its Applications to Space Mechatronics
}

\author{
Ikuo Yamamoto ${ }^{1,}$, Nobuhiro Shin ${ }^{2}$, Taishi Oka ${ }^{2}$ and Miki Matsui ${ }^{2}$ \\ ${ }^{1}$ Nagasaki University, 1-14 Bunkyomachi, Nagasaki, Nagasaki 852-8521, Japan \\ ${ }^{2}$ The University of Kitakyushu, 1-1 Hibikino, Wakamatsu, Kitakyushu, Fukuoka 808-0135, Japan \\ aiyamamoto2011@gmail.com
}

Keywords: Robotic Fish, Shark Ray, Elastic Oscillating Fin, Geological Survey, Dust Cleaning, Space

\begin{abstract}
The authors have developed a shark ray robotic fish based on biomimetic approaches. The paper describes the newly developed robotic fish technology and its application to mechatronics in the space. It is found that robotic fish technology creates not only new underwater robotics, but also the next generation space mechatronics for geological survey of lunar/planets and dust cleaning in the space station.
\end{abstract}

\section{Introduction}

Living organisms' mechanisms have evolved in order to adapt to their natural environment. The evolved mechanisms have excellent maneuvering capacities, and new robotics and machinery have often been created by implementation of biomimetic approaches [1]. The authors have developed robotic fish based on the biomimetic approaches for the next generation mechatronics. The paper describes a newly developed robotic fish technology and its application to mechatronics in the space. The authors have developed a shark ray robotic fish shown in Fig.1. The robotic fish has wing and tail structure for strong propulsion force and higher manoeuvering characteristic of turning round. The robotic fish technology can be applied to space mechatronics, such as geological survey of lunar \& planet and cleaning dust in the space station. Firstly, the mechanism of the shark ray robotic fish is explained, secondly application to geological survey mechatronics is mentioned, and thirdly that to autonomous cleaning robot for the space station is described in the paper.

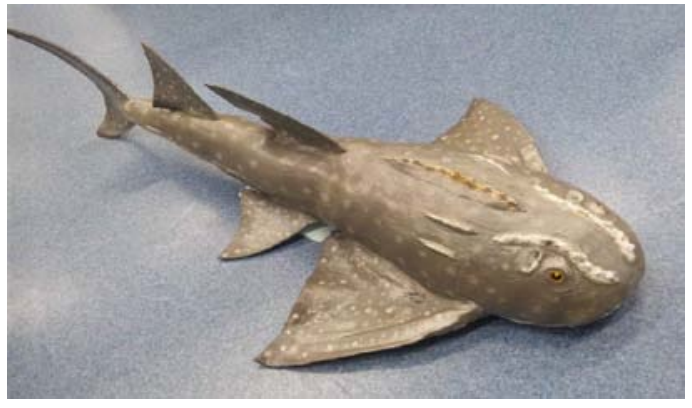

Fig. 1. Shark ray robotic fish.

\section{Robotic Fish Technology}

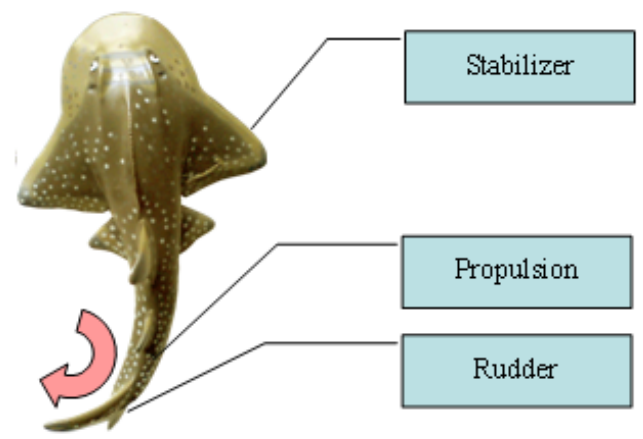

Fig. 2. Unique structure of shark ray.

The shark ray robotic fish has a unique structure. The shape of its head and wing is like a ray, and the shape of its tail is like a shark. It can act as a stabilizer, propulsion and rudder shown in Fig.2. Shark ray robotic fish has been designed by elastic oscillating fin system, which is applied to tail and main wings [2]. The robotic fish can produce the motion induced vortex at the edge of the fin [3]. Internal structure of the shark ray robotic fish is shown in Fig.3. The No.1 \& 2 motors provide propulsion force and the No.3 \& 4 motors keep motion balance in the Fig.3. The block diagram of shark ray robotic fish control system is shown in Fig.4. The control is conducted by the Arduino microcontroller board, and fins are driven by a servo motor combined with controller. The authors made experiments of thrust force for thrust performance evaluation of the robotic fish by constructing the new measuring system shown in Fig.5. The system can measure propulsion force without fixing 
the head of the robotic fish [4]. The external view of experiment system is shown in Fig.6. The robotic fish provides $60 \mathrm{~N}$ propulsion forces at $1 \mathrm{~Hz}$ of fin vibration frequency. If the frequency increases, a greater propulsion force can be provided. It has been shown that the structure of the robotic fish is effective for producing propulsion force by experiments.

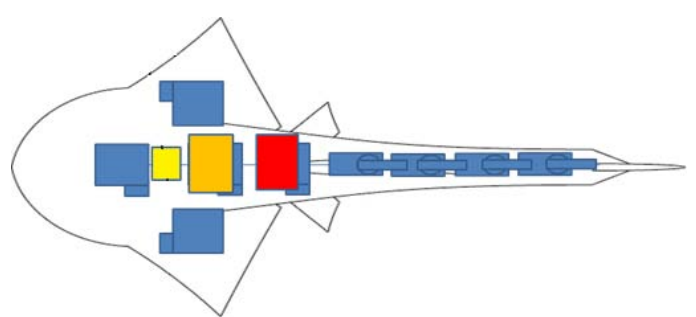

Fig. 3. Internal structure of shark ray robotic fish.

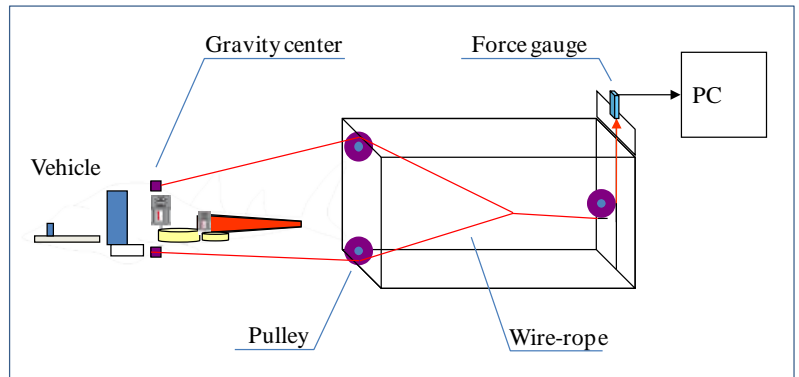

Fig. 5. Experiment system for the propulsion measurement.

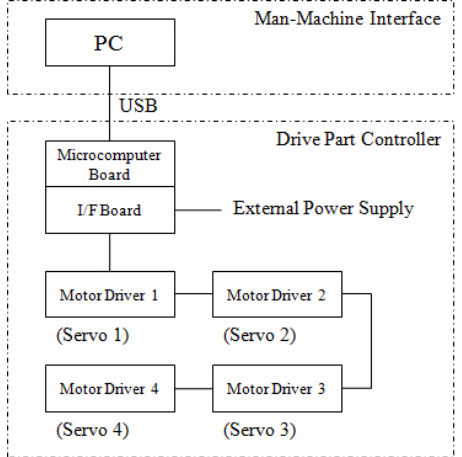

Fig. 4. Block diagram of shark ray robotic fish control system.

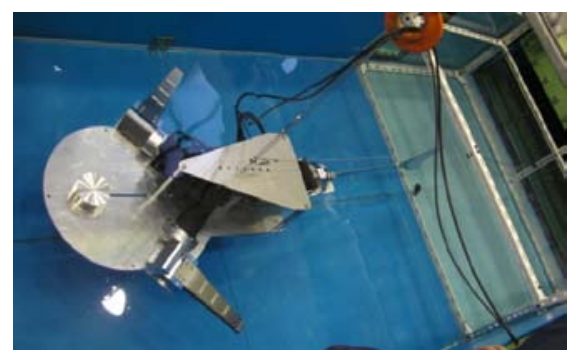

Fig. 6. The external view of experiment system.

\section{Application to Geological Survey Mechatronics}

The geological survey of lunar and planets has been planned for developing new material resources. Fig.7 shows a diagram of concept that shark ray robotic fish to survey the geological seabed. The mechanism of the robotic fish can produce the following characteristics of the wave.

Characteristics of the wave. The vibration wave (seismic waves), there is primary wave and secondary wave. Primary wave is a longitudinal wave in the elastic wave that vibrates in parallel to the traveling direction. Secondary wave is a transverse wave in the elastic wave that vibrates perpendicular to the direction of travel. Transmission velocity of primary wave and secondary wave is different from those that cause wave. Primary wave is faster than secondary wave. Secondary waves cause small tremors to arrive that to say preliminary tremors. The nature of each wave, primary wave has the characteristics of compressional wave. A change of the volume of the medium is transmitted to move. Furthermore, the wave passes through a medium such as solid, liquid or gas. On the other hand, secondary waves cannot pass through a medium that is formless as liquid or gas, because the waves transmitted by a change in the shape of the medium. Secondary wave is transmitted through the only medium of solid [5]. Currently, geological survey is investigated using the characteristics of the waves. According to the characteristics, a geological survey method takes the waveform to set up a receiver.

Geological survey method by mechatronic systems. Fig.7 is a diagram of concept that shark ray fish to survey the geological seabed. Geological survey method takes the waveform to set up a receiver to cause vibration in the shark ray robotic fish. In geological survey, the authors receive a plurality of locations to cause vibration wave in real time. The authors investigate minerals that are buried in the ground to analyze the measured waveform data. Fig.8 is a method that causes the vibration of the shark ray robotic fish. That causes secondary wave that the shark ray robotic fish is adsorbed to the seafloor and moving the tail fin. Also, it causes primary wave, which hits the ground with pectoral fins. In this case, it is assumed that the frequency of the tail fin and pectoral fins are moving at the same time. 
The authors made simulation system to verify data measurement produced by the robotic fish. The receiver shown in Fig.9 is used for the experiment that can measure the vibration of three directional compound. Fig.10 shows landscape for aligning the receiver. For experiment, the authors use vise to produce the same vibration with the robotic fish and measured 10 times while shifting the interval of distance measurement.

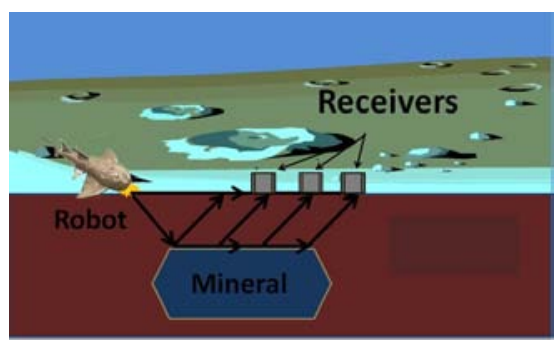

Fig. 7. Concept of lunar geological survey.

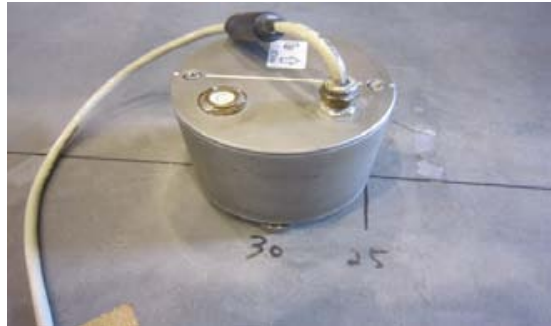

Fig. 9. Vibration measuring instrument.

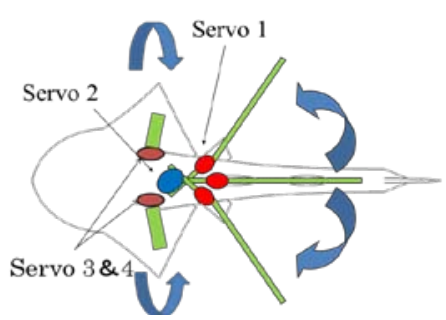

Fig. 8. Method to cause primary wave and secondary wave.

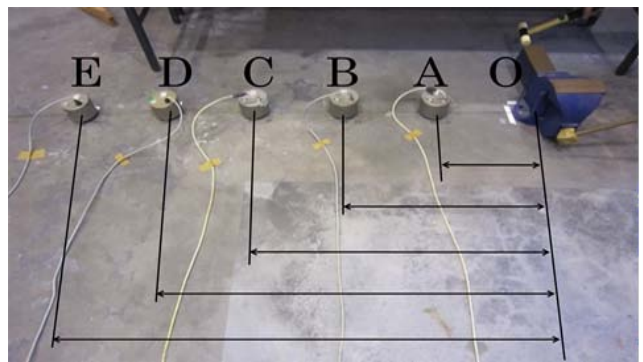

Fig. 10. Landscape for aligning the receiver.

Validation of the data. Fig.11 shows the measurement results. The measurement results of Fig.10, the horizontal axis represents time [sec], and the vertical axis represents distance $[\mathrm{m}]$. The authors raise the vibration at the position of the $t \mathrm{O}$, and measuring in the order of $\mathrm{tA}, \mathrm{tB}, \mathrm{tC}, \mathrm{tD}$, tE. For example, the authors consider the method of analysis of the experiment. In this experiment, the authors have placed the five receivers, measured the primary wave. $t A$, $t B$, $\mathrm{tC}, \mathrm{tD}, \mathrm{tE}$ is the point where vibration begins to occur in $30 \mathrm{~cm}, 150 \mathrm{~cm}, 225 \mathrm{~cm}, 300 \mathrm{~cm}, 375 \mathrm{~cm}$, respectively, from the epicenter.

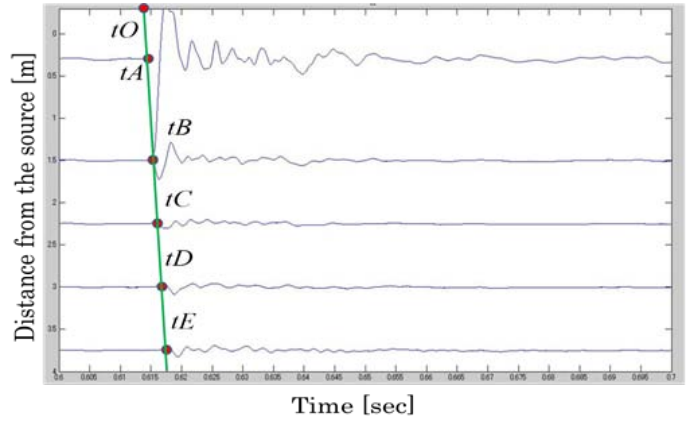

Fig. 11. Wave vibration measurements.

The authors read the time at each point because the authors don't know time tO when it began to vibration. The authors derived the time tO to use the least-square method. The authors assumed that the $y$-axis of time and the $\mathrm{x}$-axis of distance, Fig.10 shows a linear aligning described by Eq. 1.

$$
y=a x+b
$$

In addition, the authors read each point from the result that is obtained a and b by the method of least squares. The authors expressed in Eq. 2, Eq. 3 how to derive a and b of Eq. 1.

$$
a=\frac{n \sum_{i=1}^{n} x_{i} y_{i}-\sum_{i=1}^{n} x_{i} \sum_{i=1}^{n} y_{i}}{n \sum_{i=1}^{n} x_{i}^{2}-\left(\sum_{i=1}^{n} x_{i}\right)^{2}}
$$




$$
b=\frac{\sum_{i=1}^{n} x_{i}^{2} \sum_{i=1}^{n} y_{i}-\sum_{i=1}^{n} x_{i} y_{i} \sum_{i=1}^{n} x_{i}}{n \sum_{i=1}^{n} x_{i}^{2}-\left(\sum_{i=1}^{n} x_{i}\right)^{2}}
$$

According to Eq. 2, Eq. 3, the authors can be determined for each measurement tO by substituting the obtained values [6,7]. Next, the authors derive the dispersion of measurement. The authors consider that there is the dispersion due to the 10 times measurement data. The authors examine the standard deviation $\sigma$ to derive the dispersion. When set to time tO on beginning the vibration, time differences of each distance are derived $\Delta \mathrm{tA}, \Delta \mathrm{tB}, \Delta \mathrm{tC}, \Delta \mathrm{tD}, \Delta \mathrm{tE}$. For example, the authors derive the standard deviation $\sigma \mathrm{A}$ of $\Delta \mathrm{tAi}$. If the authors have $\mathrm{n}$ times measurement, determined from data $\Delta \mathrm{t} A 1, \Delta \mathrm{t} A 2$, the $\Delta \mathrm{tA} \cdots \Delta \mathrm{tAn}[6]$. Eq. 4 shows how to obtain the average value mA.

$$
m A=\frac{\Delta t A_{1}+\Delta t A_{2}+\Delta t A_{3}+\cdots \Delta t A_{n}}{n}
$$

Eq. 5 shows how to determine the standard deviation $\sigma$ A from Eq. 4.

$$
\sigma A=\sqrt{\frac{\left(\Delta t A_{1}-m A\right)^{2}+\left(\Delta t A_{2}-m A\right)^{2}+\cdots\left(\Delta t A_{n}-m A\right)^{2}}{n-1}}
$$

The authors investigated the change in waveform by comparing the values obtained. Also, if the mineral is buried under ground, the waveform considered to be changed is seen in the effect of the reflected wave caused by recoil. The authors derive the velocity of primary wave and secondary wave from these calculation methods. In addition, the Poisson's ratio $v$ for the stratum structure to examine something is derived. This is an indicator of the strain of the formation. Further, it is possible to find something in their formation. Eq. 6 shows how to calculate the Poisson's ratio.

$$
v=\frac{\left(V_{p} / V_{s}\right)^{2}-2}{2\left\{\left(V_{p} / V_{s}\right)^{2}-1\right\}}
$$

In this experiment, a value $v=0.2$ of Poisson's ratio from the calculation results were obtained. Referring to the mechanical properties, the value of the Poisson's ratio is concrete [8].

\section{Application to Cleaning Robot in the Space Station}

Environmental Control and Life Support System (ECLSS) is controlled for temperature and humidity, oxygen and carbon dioxide concentration, air-pressure, fire extinguishing, and others in the International Space Station (ISS) cabin. In addition, dust is a problem in closed cabin environment. Dust in the cabin is adsorbed in the air conditioning filter, however, it is not perfect. Astronauts using vacuum cleaner to clean dust that cannot be absorbed by air conditioning. Reducing the load of astronaut cleaning is a challenge. Method for behalf of cleaning work is required for increasing efficiency of astronauts' mission. The paper describes development of autonomous robot to clean dust in the ISS. In the study, the authors chose to fish shapes for cleaning robot. A creature suitable for movement under water has characteristics of streamlined body and fin for propulsion \& attitude control. Fish shape is excellent to transfer efficiency and maneuverability of three-dimension. Elastic oscillation fin was realized to have high controllability and soft behavior of creatures. Robotic fish is safe, because outside of robot is not equipped with rotary parts, such as propellers. The chapter describes development of autonomous robot to clean dust in the ISS. 
Overview of Robotics Fish. A creature suitable for movement under water has the characteristics streamlined body and fin for propulsion and attitude control. Therefore, fish shape is excellent to transfer efficiency and maneuverability of three-dimension. In the study, the authors choose to fish shapes for cleaning robot shown in Fig.12, using fin to move freely. Elastic oscillation fin was

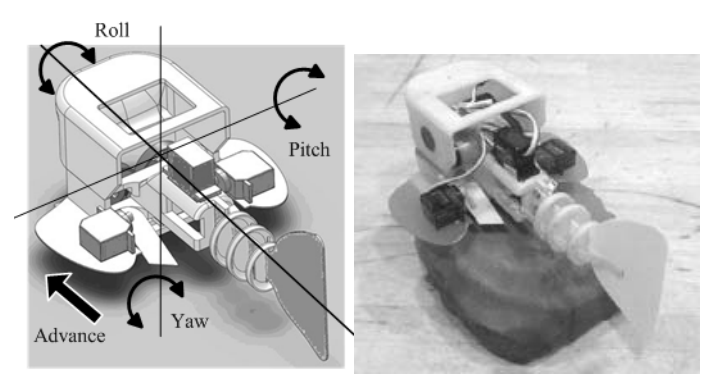

Fig. 12. Trial type robotic fish (3D CAD Prototype model). realized to have high controllability and soft behavior of creatures. Air bladder is not required for movement in zero gravity. There are many precision instruments in ISS cabin. Therefore, to detect a collision and to avoid it is required for autonomous control and maneuverability system. Also, that robotic fish does not harm is required for astronauts. Even if collision is detected, robot must be a safe shape. Robotic fish has safe mechanism. It is reason that the robot is not equipped with external rotary parts. Robotic fish takes evasive action by detecting the astronauts, walls, and obstacles. Clean robot having an autonomous flight control and navigation in Japanese Experiment Module named kibo. And, it has function to collect dust which can't be recovered by ISS air conditioning. Robotic fish starts to the autonomous navigation after a few seconds to turn on the power. Then, autonomous navigation avoids to astronauts and wall until turning on the power. Recovering dust from robotic fish is conducted act by vacuum cleaner used by astronauts. It was designed to round whale shark, which orders to calm down for astronauts. Small fish and plankton are swallowed by whale shark's wide open mouth. Likewise, dust is sucked by robotics fish's wide open mouth. Body size is a limit of legislation of less than $200 \mathrm{~mm}$ for transport by rocket. Therefore, it is required very compact movement and control mechanism. Size of the robotics fish is $199 \mathrm{~mm} \times 135 \mathrm{~mm} \times 71 \mathrm{~mm}$, total weight is about $0.2 \mathrm{~kg}$.

Outer shell of robotic fish is built by 3D printer and autonomous navigation algorithm is developed and installed in the controller [9].

Thrust and clean dust mechanism. Fig.13 represents front view of robotics fish. Main thrust is generated in two propellers to turn and counter clockwise. Rotations of propellers are countervailing to rotation moment each other. Air is aspirated by the large opening of robotics fish in the front, and breathes out to steering mechanism in the rear. Dust clean filter is equipped in front of the propellers. While cleaning performing inertial navigation, feeble negative pressure generated in the large opening of robotics fish front. Therefore, propeller to thrust is performing to collect dust. Dust clean filter is applied to absorb dust coating.

Steering mechanism. Attitude control is using the air to breathe out for robotics fish rear, and bilateral pectoral fins and caudal fin. Attitude control for the roll, pitch and yaw axis is possible by steering mechanism. It is efficient method, breathe out of air is exposed to steering mechanism directly. Elastic oscillation fin is possible to allow large curvature, then it is achieved to quick yaw axis change [10]. Elastic oscillation fin generates a small thrust from fin vibration. Fin action advances shaking the body like a fish, and is effective in search of obstacle. To stop robotics fish is turning 180 degrees in yaw axis by elastic vibration fin, and used for thrust of propeller.

Control system. In the ISS inside, LR6 batteries can use as power supply of robotics fish. Control system of robotics fish is needed for compact and low power microcomputer. Therefore, Arduino Pro Mini 328 (size: $33 \mathrm{~mm} \times 18 \mathrm{~mm} \times 3 \mathrm{~mm}$, Vcc: $3.3 \mathrm{~V}$ ) is chosen for microcomputer of robotics fish. Fig.14 represents control system of robotics fish. Start and stop of robotics fish are obeyed by power switch. Flight speed of robotics fish is controlled by volume controller. Flight speed can be set to $100 \mathrm{~mm} / \mathrm{sec}$ or less. Obstacle is detected by four infrared sensors, and to perform evasive action. These sensors arrangement is detected the direction and distance of the obstacle with respect to travelling direction. Obstacle direction is detected by infrared sensor, and moved according to the autonomous navigation algorithm. When robotics fish is collision to astronauts and obstacles, so it became to spin state. Therefore, 3-axis gyro sensor is detected for the 
angular acceleration. Robotics fish is recovered from the spin state by fins, and back to the autonomous navigation. Spin recovery operation is the highest priority in autonomous navigation.

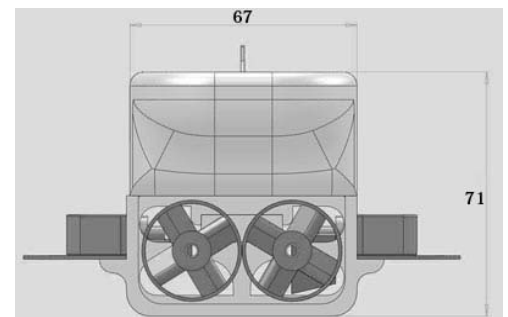

Fig. 13. Front view of robotics fish.

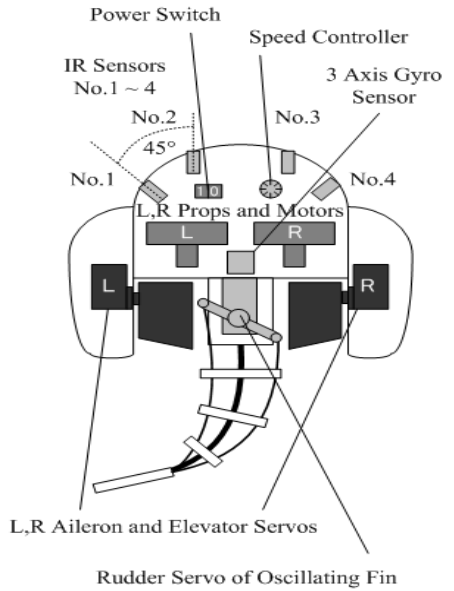

Fig. 14. Control System of Robotics Fish.

The paper has made a trial product of autonomous robotics fish to clean dust in the ISS. 3D printer can quickly manufacture parts that are designed in 3D CAD. Elastic oscillation fin was realized to have high controllability and soft behavior for creatures. It was predicted that simple algorithm of autonomous navigation is cruising the cabin almost equally to astronauts. In the future, robotics fish is required to correspondence for electro-magnetic compatibility test, vibration test, aging test, off gas test.

\section{Conclusion}

The authors have developed the robotic fish. The robotic fish technology can create new underwater robotics which can conduct a life-like swimming. It is found that the technologies of the robotic fish have much possibility for creating new space mechatronics through these applications. The authors still continue to make further experiments to produce the space products.

\section{References}

[1] Ikuo Yamamoto, Marine control systems, International Journal of Robust and Nonlinear Control, IFAC, Vol.11, No.13, Wiley, pp.1285-1341 (2001).

[2] Ikuo Yamamoto et al. Propulsion system with flexible/rigid oscillating fin, IEEE Journal of Oceanic Engineering, Vol.20, No.1, pp.23-30 (1995).

[3] Ikuo Yamamoto, Research and development of fish robot, Journal of the Mairne Engineering Society in Japan, Vol.43, No.4, pp.99-102 (2008).

[4] Ikuo Yamamoto, Tomokazu Hiratsuka, "Research and Development of Robotic Fish Based on Elastic Oscillation Fin System," Proc. of AWAS’12, Seoul, Korea, pp.1820-1834 (2012).

[5] Hiroo Kanemori, Physics of earthquake, Iwanami earth science Sensho, Iwanami Shoten, pp.26-31 (1991).

[6] Hideo Tanaka, Hisao Ishihuchi, Data analysis software, Japan Society for Fuzzy Theory and Systems Administration, Soft Computing Series, Volume 4, Asakura Shoten, pp.31-35 (1995).

[7] Kunio Miyagawa, Basic statistics Elementary Statistics [Third Edition], Yuhikaku, pp.62-67, pp.130-131 (1999).

[8] Nobuhiro Shin, Ikuo Yamamoto, Naohiro Inagawa, Kayoko Tsuruga, Daiki Makino, Research on Geology for Mechatronics systems, Proc. SICE SI 2012 (2012)

[9] Masahiko Mizui, Ikuo Yamamoto, Taishi Oka, Launch of Robotic Fish to the Space, Proc.IFAC CAMS2013 (2013).

[10] Ikuo Yamamoto, Research on an oscillating fin propulsion control system. Proc.IEEE OCEANS Vol(3), pp.259-263 (1993). 